\title{
A Review of Measuring Subjective Organization for Individuals with Brain Injuries
}

\author{
John-Christopher Finley* and Frederick Parente
}

Department of Psychology, Towson University, Towson, Maryland, USA

\begin{abstract}
Subjective organization refers to the human tendency to impose order on seemingly unrelated events in a person's environment. This phenomenon that has been studied among individuals with acquired and traumatic brain injuries as they have a diminished ability to subjectively organize novel information. Thus, researchers have begun developing measures of subjective organization intended to be used as a diagnostic tool. The purpose of this review is to summarize what is known about subjective organization and to outline how a measure of subjective organization can be used to assess the extent of organizational deficits for persons with acquired and traumatic brain injuries. Moreover, this review will discuss the clinical implications of measuring subjective organization among individuals with a brain injury.
\end{abstract}

Keywords: Subjective organization; Brain injury; Word recall; Negative transfer; Positive transfer

\section{Introduction}

Subjective organization (SO) refers to the human tendency to impose order on seemingly unrelated events in a person's environment [1]. Tulving [2] and Miller [3] were among the first to observe that people organize verbal information, such as word lists, into higher order units, usually consisting of two or more related words. Miller [3] speculated that this organization process underlies retention and recall of everyday information. The extent to which a person forms SO units is directly related to recall. Given that SO mediates learning, it is important to have a means of assessing the SO process. An effective measurement procedure could be especially valuable when assessing persons who have diminished ability to organize, e.g., those with traumatic brain injury (TBI) or stroke [1,4-6]. Until recently, however, there has been a dearth of research that has directly measured SO among individuals with an acquired brain injury $[7,8]$.

Perhaps the first measures of SO appeared in the 1970's [8]. However, these measures were designed to assess clustering of unrelated word lists in multi-trial free recall learning with college students. The indices examined the extent to which college students would recall word pairs over sequential trials. Since the mid-70s, there have been only a handful of published research that has addressed the topic of $\mathrm{SO}[2,6,9]$. The purpose of this review is to summarize what is known about SO and to outline how a measure of $\mathrm{SO}$ can be used to assess the extent of organizational deficits for persons with TBI or stroke.

\section{Theoretical Perspectives}

Frequency theory [10-12] asserts that frequency of exposure to novel information facilitates recall. Sternberg and Tulving [8] reported that, in addition to increased frequency of word exposure, a person's uniquely developed patterns of word recall determined the rate of learning of the words. This unique sequential ordering of words during the study/test sequence became more prominent as learning progressed. Thus, Tulving [2] argued that mere repetition of exposure to novel events in our environment is insufficient for successful recall; rather the rate that a person learns verbal information depends on his or her ability to organize the information in a unique manner. Tulving's [2] premise has been supported by other studies $[13,14]$.

\section{Measurement of SO}

Several researchers have attempted to demonstrate the effect of $\mathrm{SO}$ on the learning process. However, most studies involve verbal learning typically with unrelated word lists. One common method for demonstrating the effect of SO on learning involves either a "partwhole" transfer paradigm $[2,6,9]$ or a "whole-whole" transfer paradigm. The part-whole paradigm begins with a training phase where an experimental and control group initially learn different lists of unrelated words (part-list) that are presented individually in randomized order over a multi-trial learning sequence. Each group then learns a second list which is twice as long as the first. However, the experimental group participants' second list (whole-list) of words contains the entire first list plus an equal number of new unrelated words. The control group participants' second list includes twice as many new words none of which overlap with the first list. This paradigm can be used to demonstrate a counterintuitive negative transfer of learning effect. Because the experimental group initially learned half of the second list words, it is reasonable to suggest that they would learn the second list faster than the control group. However, just the opposite effect occurs. That is, performance of the experimental group is better than the control on the initial second list trials but the participants in this group begin experiencing organizational interference and their performance is soon surpassed by the control group participants.

\section{Negative transfer}

Tulving [2] was the first to show that during second list learning, the experimental group participants demonstrate slower learning relative to the control group. This phenomenon has been demonstrated in a number of subsequent studies [9]. Tulving [2] speculated that

*Corresponding author: John-Christopher Finley, Department of Psychology Towson University, 8000 York Rd. Towson, Maryland, 21252, USA, Tel: +(215) 589-9053; E-mail: jfinle2@students.towson.edu

Received: December 07, 2017; Accepted: December 15, 2017; Published: December 22, 2017

Citation: Finley JC, Parente F (2017) A Review of Measuring Subjective Organization for Individuals with Brain Injuries. Clin Exp Psychol 3: 176. doi: 10.4172/2471-2701.1000176

Copyright: ( 2017 Finley JC, et al. This is an open-access article distributed under the terms of the Creative Commons Attribution License, which permits unrestricted use, distribution, and reproduction in any medium, provided the original author and source are credited. 
the experimental group participants subjectively organized the words during first list learning and this SO had to be reorganized during second list learning which created negative transfer. The finding was counterintuitive because the experimental group participants had already learned half of the words which should have, according to the frequency theory, improved their rate of learning of the second list items.

The whole-whole transfer of learning paradigm (a variation of the part-whole paradigm) has been shown to produce similar results. In this procedure, participants learn two lists of words that are of equal length. However, the second list contains half of the first list words and half new words. Both lists are repeated over a multi-trial learning task after which the old and new words from the second list are plotted. Several studies have shown that the rate of learning for the new words is significantly greater than it is for the old words.

The whole-whole procedure was developed initially as a clinical tool. Because an individual learns both old and new words during second list learning, it is possible to plot his or her performance over trials for each word type. Differences in the learning curves for each type of word is then used as an index of the interfering effects of SO on the learning process. For example, inhibited recall of the old words relative to the new on the second list suggests that the person subjectively organized the old words during first list learning, whereas positive transfer of the old words relative to the new suggests a lack of SO. Because this task can be used to demonstrate the presence or absence of SO with individual participants, Wilbur et al. [9] suggested that it could be used as a performance based index of SO with clinical populations. Moreover, the technique could be used to measure SO with any type of learning materials (e.g., shapes rather than words).

\section{Non-verbal SO}

With the exception of two unpublished studies [15,16], SO using the part-whole or whole-whole paradigm has been studied only using word lists. Thus, it was unknown whether individuals subjectively organize non-verbal information. These authors used a whole-whole learning paradigm with verbal and non-verbal (symbols) stimulus items. Both studies replicated the familiar negative transfer effect with word lists but showed positive transfer with shape lists. Although it is reasonable to suggest that $\mathrm{SO}$ is less prominent with non-verbal information, the question remains unanswered.

\section{Performance-based measures of SO}

Organizational deficits are typically inferred from observations of everyday behavior [17]. There are, however, no commonly used measures of SO performance from which a clinician can assess a person's ability to organize. This type of measure would have to derive from a numerical scoring of a person's ability to perform a task. Sternberg and Tulving [8] proposed that any such measure of SO must include the following psychometric properties: quantification, reliability, construct validity, and empirical validity. Thus, a measure of SO must be quantifiable with a numerical scale, demonstrate reproducible scoring, measure what it claims to measure, and correlate predictably with other measures to which they are theoretically related. Sternberg and Tulving [8] described several candidate assessments that could possibly measure SO for verbal information; however, these measures only index sequential association of adjacent recall of unrelated words $[8,18]$. Moreover, none of the indices were intended for use as a clinical tool.

Parente and Finley [7] proposed a performance-based measure of
SO that is more versatile than those that were evaluated by Sternberg and Tulving [8]. The analysis involved assessing Association Rules that derived from a person's total recall across multiple learning trials. Association Rules (AR) analysis is commonly used in the business world to assess the purchase patterns of consumers in marketing studies. Parente and Finley [7] applied the same analysis to individual's recall of words across a multi-trial learning sequence to compute an index of association for the various words in an unrelated word list. The analysis provided a number of different measures of organization, some of which correlated with the persons' recall. The AR index conformed to all of the constraints outlined by Sternberg and Tulving [8] and it had the additional advantage of measuring organization globally rather than over sequential trials.

Parente and Finley [7] suggested that one of the AR measures (Confidence) was the best candidate for an index of SO. The confidence index measures the joint probability of recalling, for example, item $\mathrm{B}$ given that the person also recalls item $\mathrm{A}$. The confidence computations assess associations among any items that occur together consistently during learning without having, necessarily, to be recalled adjacent to one another. Additionally, these measures of association rules can be computed using commercially available software. Furthermore, association rule measures can be applied to any type of recall or item generation, including recall of visual information. Parente and Finley [7] found that the confidence AR measure was highly correlated with recall which suggests that it may be of use as a convenient adjunct tool in any commercially available multi-trial learning task (e.g., California Verbal Learning Test).

\section{Clinical Implications}

All of the studies discussed above suggest that SO mediates learning $[1,6]$. However, because $\mathrm{SO}$ is subjective, there have been limited means to measuring it directly. Most studies use indirect measures that rely on observational data or inference based on patterns of scores from psychological tests $[4,5]$. It is commonly accepted that brain injury or stroke reduces a person's ability to organize $[19,20]$. It is, therefore, necessary to develop a sensitive, objective, and performance based measure of organization that can be used to assess baseline SO skill and to document improvement over time. It is evident that $\mathrm{SO}$ gives insight into a persons' capability to learn and memorize novel information; but it may also provide information regarding a patient's executive functioning. Executive function refers to self-regulatory functions that organize and direct cognitive activity, emotional response, and overt behavior [5]. More importantly, executive dysfunction often limits a person's ability to organize information [5], which likely interferes with a persons' ability to subjectively organize.

\section{Practicalities}

How does one actually measure $\mathrm{SO}$ using the techniques described above? Using the Whole-Whole transfer approach, the clinician would first construct two lists of unrelated nouns; each list is of equal length. The second list would include half of the words from the first. We have found that with brain injured patients, each list should contain no more than 12 words. The patient learns the first list over at least six test trials study-test trials whereby the clinician reads the words of either list individually at two second intervals and in a random order on each trial. The measure of performance is the number of recalls on each trial. This same study-test sequence is repeated with the second list after which the clinician plots the number of new and overlapping (old) words separately that were recalled across the study-test sequence $[21,22]$. 
Citation: Finley JC, Parente F (2017) A Review of Measuring Subjective Organization for Individuals with Brain Injuries. Clin Exp Psychol 3: 176. doi: $10.4172 / 2471-2701.1000176$

Page 3 of 3

Typically, three patterns of performance can emerge in the plot of the second list old and new words. One type of patient will demonstrate reduced learning of the old words relative to the new. This pattern suggests that the person subjectively organized the overlapping words during first list learning and their acquired SO inhibited recall of the words on the second list. A second pattern of performance shows that the old words are recalled better than the new words on the second list. This pattern indicates that the person benefited from frequency of exposure for the old words on the first list but did not demonstrate any evidence of SO. The third pattern illustrates no difference between the old and new word recall on the second list. This pattern suggests that the person lacks awareness that the old words occurred again on the second list.

The first pattern suggests that the patient can organize. It might also suggest less of a cognitive deficit, or improvement in organizational skill with treatment or simply the passage of time. The second pattern suggests that the patient does not organize effectively or perhaps has experienced little improvement in organization relative to an earlier measurement or after treatment. The third pattern indicates a rather severe lack of awareness which often results from a global executive dysfunction.

The second approach to measuring SO involves computing an actual measure of organization. We have adapted association rule analysis for this purpose which requires use of computer software for computing the various measures. These measures are available from different vendors, e.g., SPSS modeler, KHCoder, or Magnum Opus. To begin, the clinician would simply present a list of random nouns (e.g., 12 words) in random order and ask the patient to recall the words over six or more individual trials. The clinician writes down each recalled word on each trial then enters the words into a spreadsheet and submits the entirety of the persons' word recall data to any of the software packages listed above. The analysis provides a variety of measures that index the joint probability of recall for word pairs and larger associated word sequences. We have found that the confidence measure provided by this analysis is the most sensitive index of SO. In addition, the number of word associations and the complexity of the sequences are also highly correlated with recall and can be used as indices of SO.

\section{Limitations and Implications}

This line of research is still in it's infancy. Although the measures of SO discussed above have been used informally with our clinical populations, there are few studies that have validated these measures with large populations. There are no published norms for the measures and no standardized administration procedures. There are no studies of clinical populations, use of the measures with visual information, or studies of organization in recognition memory. At present, they are best used as adjunct analyses for any test that involves a multi-trial study/test recall sequence. For example, the California Verbal Learning Test uses a word recall task that is administered repeatedly over trials. The AR measure might be computed across the learning sequence to provide useful additional information about the person's ability to subjectively organize.

\section{References}

1. Parente R, Herrmann D (2003) Retraining working memory. In Retraining cognition: Techniques and applications (2nd edn.). Pro-Ed, Austin, TX. pp: 121-129.

2. Tulving $E$ (1966) Subjective organization and effects of repetition in multi-trial free-recall learning. J Verbal Learning Verbal Behav 5: 193-197.

3. Miller GA (1956) The magical number seven, plus or minus two: some limits on our capacity for processing information. Psychol Rev 63: 81-97.

4. Levin HS, Culhane KA, Mendelsohn E, Lilly MA, Bruce D, et al. (1993) Cognition in relation to magnetic resonance imaging in head-injured children and adolescents. Arch Neurol 50: 897-905.

5. Gioia GA, Isquith PK (2004) Ecological assessment of executive function in traumatic brain injury. Dev Neuropsychol 25: 135-158.

6. Twum M (1994) Maximizing generalization of cognitions and memories after traumatic brain injury. Neuro Rehabilitation 4: 157-167.

7. Parente R, Finley JC (2017) Using association rules to measure subjective organization after acquired brain injury. Neurorehabilitation.

8. Sternberg RJ, Tulving E (1977) The measurement of subjective organization in free recall. Psychol Bull 84: 539-556.

9. Wilbur R, Silver R, Parente R (2007) Subjective organization and positive transfer of performance with traumatically brain-injured adults. J Clin Exp Neuropsychol 29: 682-689.

10. Bousfield WA, Cohen BH (1955) The occurrence of clustering in the recall of randomly arrayed words of different frequencies of usage. J Gen Psychol 52: 83-95.

11. Deese $J(1960)$ Frequency of usage and number of words in free recall: The role of association. Psychol Rep 7: 337-344.

12. Postman $L$ (1970) Effects of word frequency on acquisition and retention under conditions of free-recall learning. Q J Exp Psychol 22: 185-195.

13. Koriat A, Melkman R (1981) Individual differences in memory organization as related to word association, object sorting, and word matching styles. $\mathrm{Br} J$ Health Psychol 72: 1-18.

14. Mandler G (1967) Organization and memory. Psychol Learn Motiv 1: 327-372.

15. DeMatt $E$ (2009) Subjective organization in verbal versus visual memory tasks Unpublished manuscript, Towson University, Towson, Maryland.

16. Nickerson SD (2014) An experimental investigation of subjective organization in college students and traumatically brain injured individuals. Towson University Institutional Repository, Towson, Maryland.

17. Gioia GA, Isquith PK, Guy SC, Kenworthy L (2000) Test review behavior rating inventory of executive function. Child Neuropsychol 6: 235-238.

18. Senkova O, Otani H (2015) Subjective organization calculator for free recall. SAGE Open 5: 2158244015615637.

19. Cullum CM, Kuck J, Ruff RM (1990) Neuropsychological assessment of traumatic brain injury in adults. In: Bigler (ed.), Traumatic brain injury: Mechanisms of damage, assessment, intervention, and outcome. Pro-Ed Austin, TX. pp: 129-163.

20. Schacter DL, Crovitz HF (1977) Memory function after closed head injury: A review of the quantitative research. Cortex 13: 150-176.

21. Dienes Z, Berry D (1997) Implicit learning: Below the subjective threshold Psychon Bull Rev 4: 3-23.

22. Tulving E (1962) Subjective organization in free recall of "unrelated" words Psychol Rev 69: 344-354. 